WM-06-104

\title{
Unusual High-Energy Phenomenology of Lorentz-Invariant Noncommutative Field Theories
}

\author{
Christopher D. Caront* and Herry J. Kweđt \\ Particle Theory Group, Department of Physics, \\ College of William and Mary, Williamsburg, VA 23187-8795
}

(Dated: March 2006)

\begin{abstract}
It has been suggested that one may construct a Lorentz-invariant noncommutative field theory by extending the coordinate algebra to additional, fictitious coordinates that transform nontrivially under the Lorentz group. Integration over these coordinates in the action produces a fourdimensional effective theory with Lorentz invariance intact. Previous applications of this approach, in particular to a specific construction of noncommutative QED, have been studied only in a lowmomentum approximation. Here we discuss Lorentz-invariant field theories in which the relevant physics can be studied without requiring an expansion in the inverse scale of noncommutativity. Qualitatively, we find that tree-level scattering cross sections are dramatically suppressed as the center-of-mass energy exceeds the scale of noncommutativity, that cross sections that are isotropic in the commutative limit can develop a pronounced angular dependence, and that nonrelativistic potentials (for example, the Coloumb potential) become nonsingular at the origin. We consider a number of processes in noncommutative QED that may be studied at a future linear collider. We also give an example of scattering via a four-fermion operator in which the noncommutative modifications of the interaction can unitarize the tree-level amplitude, without requiring any other new physics in the ultraviolet.
\end{abstract}

* carone@physics.wm.edu

†hxjohn@wm.edu 


\section{INTRODUCTION}

Noncommutative field theories have provoked considerable interest from both the for-

mal [1] and phenomenological [2] perspectives. In their simplest construction, such theories promote spacetime coordinates to operators (indicated below by a hat) that satisfy a nontrivial commutation relation

$$
\left[\hat{x}^{\mu}, \hat{x}^{\nu}\right]=i \theta^{\mu \nu}
$$

where $\theta^{\mu \nu}$ is an antisymmetric, constant matrix. The noncommutative multiplication of operators is represented via function of ordinary, commuting coordinates by means of a modified multiplication rule, the star product

$$
f(x) \star g(x)=f(x) e^{\frac{i}{2} \overleftarrow{\partial} \cdot \theta \cdot \vec{\partial}} g(x)
$$

For example, it is easy to show that the commuting coordinates $x^{\mu}$ satisfy the star commutation relation

$$
\left[x^{\mu} \stackrel{\star}{,} x^{\nu}\right]=i \theta^{\mu \nu}
$$

mimicking the behavior of the operators in Eq. (1.1). Noncommutative fields theories are related to their commutative cousins via the promotion of ordinary multiplication to star multiplication, and the imposition of restrictions on the form of the interactions as needed to maintain the desired local symmetries of the theory [3]].

The effects of noncommutative spacetime on rare processes [4], collider signals [5], astrophysics [6], and cosmology [7] have been discussed in the literature over the past few years. One notable feature of noncommutative field theories based on Eq. (1.1) is that they violate Lorentz invariance; despite its appearance, the matrix $\theta^{\mu \nu}$ is a singlet under the Lorentz group, and defines preferred the directions $\epsilon^{i j k} \theta^{j k}$ and $\theta^{0 i}$. This has led to the observation that collider signatures of noncommutative physics should present a diurnal variation, as the earth's orientation relative these preferred directions varies over time [8]. On the other hand, noncommutative field theories must contend with very stringent bounds from processes measured precisely at low energies [9]. For example, in almost every construction of Lorentz-violating noncommutative QED, there are contributions to the Lamb shift which force the scale of new physics to be above $\sim 10 \mathrm{TeV}$ 10]. Moreover, in the simplest formulation of noncommutative gauge theories (for example, the version of noncommutative QED 
first introduced by Hayakawa [1]]), one may show that operators induced at the one-loop level present even more stringent bounds on the noncommutative scale, whether the loops are evaluated with a simple cut-off or a gauge-invariant regulator 12, 13, 14]. Noncommutative gauge theories based on the enveloping algebra approach 15] contribute to the Lamb shift at tree-level, and are thus subject to the $10 \mathrm{TeV}$ bound. These theories may also be constrained significantly via loop-induced operators [16], though in this case the situation is less clear [17]. However one views the severity of these bounds, it is clear in any construction of noncommutative field theories based on Eq. (1.1), the matrix $\theta^{\mu \nu}$ will appear in the lowenergy effective Lagrangian as a Lorentz-violating spurion. By contrast, all experimental searches for the violation of Lorentz invariance have yielded negative results to date [18, 19].

These observations provide some motivation for considering whether noncommutative theories can be constructed which preserve Lorentz invariance $a b$ initio $^{1}$. In this paper, we will revisit a proposal made in Ref. [21] (and studied in Refs. [22, 23, 24, 25, 26]) which approaches this problem by promoting $\theta^{\mu \nu}$ to an operator in Eq. (1.11),

$$
\left[\hat{x}^{\mu}, \hat{x}^{\nu}\right]=i \hat{\theta}^{\mu \nu}
$$

where $\hat{\theta}^{\mu \nu}$ transforms as a tensor under the Lorentz group. In addition, one assumes the simplest possibility $\left[\hat{x}^{\mu}, \hat{\theta}^{\alpha \beta}\right]=\left[\hat{\theta}^{\mu \nu}, \hat{\theta}^{\alpha \beta}\right]=0$. The star product for this algebra takes the same form as Eq. (1.2), except that it acts, in general, on functions of six additional commuting coordinates $\theta^{\mu \nu}$

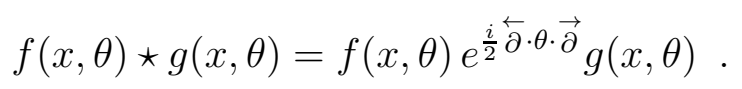

As argued in Ref. [21], the action will involve integration over the additional coordinates

$$
\mathcal{L}_{\text {eff }}=\int d^{6} \theta W(\theta) \mathcal{L}\left(x^{\rho}, \theta^{\mu \nu}\right)
$$

so that $\mathcal{L}_{\text {eff }}$ depends only on ordinary coordinates and has four-dimensional Lorentz invariance preserved. The weighting function $W(\theta)$ is a Lorentz-invariant function of $\theta^{\mu \nu}$ that has finite volume and hence drops off at large values of the argument. In the mapping from the

\footnotetext{
${ }^{1}$ An alternative approach is to construct a noncommutative theory that preserves a deformation of the Lorentz group [20]. For other discussions of Lorentz symmetry in noncommutative theories, see Refs. [27, 28]
} 
space of operators to c-number functions, it was argued in Ref. [21], that the $d^{6} \theta W(\theta)$ integration is an appropriate mapping of the operator trace, which is reasonable to believe plays a role in the construction of an action. In the present context, it is only important to note that $W(\theta)$ determines the scale of the new physics. For example, the choice $W(\theta)=\delta^{(6)}(\theta)$ pushes the scale of noncommutativity to infinity.

Two question deserve immediate comment: (1) Is there anything wrong with treating $\theta^{\mu \nu}$ as a set of fictitious coordinates with nontrivial Lorentz transformation properties and (2) where do ordinary quantum fields live within the right-hand-side of Eq. (1.6)?

On the first question, it is useful to consider the Grassman parameters $\theta$ and $\bar{\theta}$ of ordinary superspace. These may be thought of as fictitious coordinates that transform nontrivially under the Lorentz group and are integrated in the action to produce a Lagrangian that is invariant under global supersymmetry transformations. Extending the coordinate algebra by the bosonic coordinates $\theta^{\mu \nu}$ in Eq. (1.6) is neither more nor less justified mathematically then extending ordinary space to superspace. On the other hand, Doplicher, Fredenhaeghen and Roberts [29] have argued on general grounds that theories of quantum gravity should lead at low energies to spacetime noncommutativity described by a commutation relation of precisely the same form as Eq. (1.4), one in which the right-hand-side is an operator that transforms nontrivially under the Lorentz group so that the result remains covariant ${ }^{2}$.

On the second question, we may assume that the Lagrangian $\mathcal{L}$ is a function of fields $\Phi$ that are themselves functions of $x^{\mu}$ and $\theta^{\mu \nu}$, with all multiplications between fields promoted to star multiplications. In the supersymmetric analogy, superfields are functions of all the superspace coordinates, while ordinary fields appear in the coefficients of an expansion of the superfields in $\theta$ and $\bar{\theta}$. It was shown in Ref. 21] that an analagous statement can be made for the fields $\Phi\left(x^{\rho}, \theta^{\mu \nu}\right)$

$$
\Phi(x, \theta)=\phi(x)+\theta^{\mu \nu} \Phi_{\mu \nu}^{(1)}(x)+\theta^{\mu \nu} \theta^{\eta \sigma} \Phi_{\mu \nu \eta \sigma}^{(2)}(x)+\cdots,
$$

where the $\Phi^{(n)}$ are functions of the ordinary quantum field $\phi(x)$. (Of course, unlike the supersymmetric example, the expansion here does not truncate.) In Ref. 21], it was shown that the constraint of gauge invariance allows one to determine the $\Phi^{(n)}$ as functions of the

\footnotetext{
${ }^{2}$ One can also understand the algebra of interest to us here as a contraction of the one proposed by Snyder [30]. See Ref. 21] for details.
} 
quantum field $\phi$ and the gauge fields in the theory. This allowed for the construction of a Lorentz-invariant version of noncommutative QED, one that was valid for fields of arbitrary charge.

One of limitation of the models discussed in Refs. [21, 25] is that they could only be evaluated as an expansion in $\theta$ (or more accurately, after the $d^{6} \theta$ integration, as an expansion in momentum divided by the typical noncommutative energy scale). The phenomenological study of Lorentz-invariant noncommutative QED in Ref. 25] focused, therefore, on the effects of the new higher-dimension operators and presented bounds on a natural definition of the noncommutative scale

$$
\Lambda_{N C}^{-4}=\frac{1}{12} \int d^{6} \theta \theta^{\mu \nu} \theta_{\mu \nu} W(\theta)
$$

An advantage of this approach is that the form of the weighting function $W(\theta)$ need not be specified, at least for lowest-order processes. Comparison of Bhabha scattering, dilepton and diphoton production to LEP data led the authors of Ref. 25] to the bound

$$
\Lambda_{N C}>160 \mathrm{GeV} \quad 95 \% \text { C.L. }
$$

The purpose of the present work is to consider toy and more realistic examples where Lorentz-invariant noncommutative theories can be evaluated without a low-momentum expansion. The toy models will consist of the Yukawa and $\phi^{4}$ theories. Since the expansion in Eq. (1.7) is not restricted by gauge invariance in these theories, there is no inconsistency in making the simplest choice that might allow us to obtain results in closed form

$$
\Phi(x, \theta)=\phi(x)
$$

The more realistic theory that we will study is the original formulation of noncommutative QED [11], which also does not require a $\theta$-expansion for the fields, at the expense of introducing a restriction that the matter fields have charges \pm 1 or 0 . This will allow us to revisit the purely leptonic processes considered in the low-momentum limit of the alternative formulation discussed in Ref. [25]. Finally, to avoid an expansion in $\Lambda_{N G}^{-1}$, we will have to choose a tractable form for the weighting function $W(\theta)$. Neither Ref. [21] or [22, 23, 24, 25, 26] ever specify $W(\theta)$, or show that a function with the desired properties exists. We will find the simplest form for this function, based on the quadratic invariants of $\theta^{\mu \nu}$, that will allow for explicit calculations at arbitrary external momenta. 
Our paper is organized as follows. In Section II] we show that suitable weighting functions exist, and we determine a simple form that will be used in evaluating the models that follow. In Section [II] we will look at the behavior of tree-level scattering cross sections in $\phi^{4}$ and Yukawa theory. We will see that cross sections experience a notable suppression as the center-of-mass energy exceeds the typical scale of the noncommutative interactions and that isotropic, tree-level processes in the commutative limit can develop a marked angular dependence. In Section IV] we will extend our analysis to noncommutative QED, focusing in particular on Bhabha scattering, dilepton and diphoton production. We will also show that the modified momentum dependence of the tree-level fermion-photon vertex removes the singularity at the origin of the Coloumb potential. In Section [V] we show that a simple theory that violates tree-level unitarity in the commutative limit can be be unitarized by the noncommutative modification to the vertex. In Section VI, we summarize our conclusions.

\section{THE WEIGHTING FUNCTION}

In this section, we find a suitable explicit form for the weighting function $W(\theta)$ in Eq. (1.6). In particular, we would like $W(\theta)$ to be Lorentz invariant and normalizable [21]. If the new coordinates carried only a single Lorentz index (i.e., if they transformed like the ordinary coordinates $x^{\mu}$ ) it would not be possible to find such a function: All Lorentzinvariant functions of $x^{\mu}$ are necessarily a function of $x^{\mu} x_{\mu}$, which vanishes along its light cone. Thus, integration over all four-volume would diverge at large $x^{\mu}$ in the direction where $x^{\mu} x_{\mu}=0$.

As pointed out by Kase, et al., the same is true of weighting functions $W(\theta)$ that are

only functions of $\theta^{\mu \nu} \theta_{\mu \nu}$ [23]. One approach, taken in Ref. [23], is to use a very manageable function, for example a Gaussian in $\theta^{\mu \nu} \theta_{\mu \nu}$, and regulate away the unwanted divergence. Here we will show that simple weighting functions $W(\theta)$ exist that have finite volume and are not so complicated that they render explicit calculations intractable. In contrast to a coordinate with a single Lorentz index, $\theta^{\mu \nu}$ allows for the construction of an infinite number of Lorentz invariant combinations, for example

$$
\theta^{\mu \nu} \theta_{\mu \nu}, \quad \theta^{\mu \nu} \theta_{\nu \rho} \theta^{\rho \alpha} \theta_{\alpha \mu}, \quad \theta^{\mu \nu} \theta_{\nu \rho} \theta^{\rho \alpha} \theta_{\alpha \beta} \theta^{\beta \delta} \theta_{\delta \mu}, \quad \text { etc. }
$$

In general, the directions in which these invariants are vanishing differ from one to another. 
Thus, we seek a function that drops off at large values of all of its arguments and that depends on a sufficient number of these invariants so that no flat directions remain.

The simplest possibility is to work only with the two available quadratic invariants

$$
\frac{1}{2} \theta_{\mu \nu} \theta^{\mu \nu} \text { and } \frac{1}{8} \epsilon_{\mu \nu \alpha \beta} \theta^{\mu \nu} \theta^{\alpha \beta},
$$

where the numerical factors are introduced for later convenience. To make integration as easy as possible, we choose to work with an exponential form for $W(\theta)$. One might begin by considering the function

$$
W_{b c}(\theta) \equiv \exp \left(-\frac{c}{2}\left|\theta_{\mu \nu} \theta^{\mu \nu}\right|\right) \exp \left(-\frac{b}{8}\left|\epsilon_{\mu \nu \alpha \beta} \theta^{\mu \nu} \theta^{\alpha \beta}\right|\right),
$$

where $b$ and $c$ are constants, and the absolute value symbols are introduced to assure that there are no directions in which the weighting function blows up at infinity. Unfortunately, the function $W_{b c}(\theta)$ is not adequate since integration over all $\theta^{\mu \nu}$ leads to a divergent result. However, by studying this divergence we will see that there is a simple way to avoid it.

Let us parameterize the components of $\theta^{\mu \nu}$ as follows:

$$
\theta^{\mu \nu}=\left(\begin{array}{cccc}
0 & x & y & z \\
-x & 0 & u & -v \\
-y & -u & 0 & w \\
-z & v & -w & 0
\end{array}\right) .
$$

In terms of these variables, the two lowest-order, Lorentz invariant forms can be written as:

$$
\begin{aligned}
\frac{1}{2} \theta_{\mu \nu} \theta^{\mu \nu} & =-x^{2}-y^{2}-z^{2}+u^{2}+v^{2}+w^{2}, \\
\frac{1}{8} \epsilon_{\mu \nu \alpha \beta} \theta^{\mu \nu} \theta^{\alpha \beta} & =x w+y v+z u .
\end{aligned}
$$

By inspection, the right-hand sides of Eq. (2.5) take a simple form if we redefine our variables again, in terms of two sets of spherical polar coordinates:

$$
\begin{aligned}
& (x, y, z) \rightarrow\left(r_{1}, \theta_{1}, \phi_{1}\right), \\
& (w, v, u) \rightarrow\left(r_{2}, \theta_{2}, \phi_{2}\right) .
\end{aligned}
$$

The function $W(\theta)_{b c}$ can now be written

$$
W(\theta)_{b c}=\exp \left(-c\left|r_{1}^{2}-r_{2}^{2}\right|\right) \exp \left(-b\left|\overrightarrow{r_{1}} \cdot \overrightarrow{r_{2}}\right|\right) .
$$


The $d^{6} \theta \equiv d^{3} r_{1} d^{3} r_{2}$ integration is now easy to evaluate since Eq. (2.7) depends only on $r_{1} \equiv\left|\overrightarrow{r_{1}}\right|, r_{2} \equiv\left|\overrightarrow{r_{2}}\right|$ and the angle between $\overrightarrow{r_{1}}$ and $\overrightarrow{r_{2}}$. Furthermore, one may do the $r_{2}$ integration first, letting the $\overrightarrow{r_{1}}$ direction define the $z$-axis, so that the angular integration simplifies to $\int d \Omega_{1} d \Omega_{2}=\int 8 \pi^{2} \cdot d \cos \theta_{2}$. One then finds that

$$
\int d^{6} \theta W(\theta)_{b c}=\frac{16 \pi^{2}}{b} \int d r_{1} r_{1} \int d r_{2} r_{2} \exp \left(-c\left|r_{1}^{2}-r_{2}^{2}\right|\right)\left[1-\exp \left(-b r_{1} r_{2}\right)\right] .
$$

The $\int d r_{1} r_{1} \int d r_{2} \cdot r_{2} \exp \left(-c\left|r_{1}^{2}-r_{2}^{2}\right|\right)$ integration is divergent, while the rest is finite. However, this contribution also scales as $1 / b$, while the finite part does not, suggesting an alternative starting point in which this divergence is cancelled

$$
W(\theta)=N\left[W(\theta)_{a c}-\frac{b}{a} W(\theta)_{b c}\right]
$$

or explicitly,

$$
\mathrm{W}(\theta)=N \exp \left(-\frac{c}{2}\left|\theta_{\mu \nu} \theta^{\mu \nu}\right|\right)\left[\exp \left(-\frac{a}{8}\left|\epsilon_{\mu \nu \alpha \beta} \theta^{\mu \nu} \theta^{\alpha \beta}\right|\right)-\frac{b}{a} \exp \left(-\frac{b}{8}\left|\epsilon_{\mu \nu \alpha \beta} \theta^{\mu \nu} \theta^{\alpha \beta}\right|\right)\right],
$$

where $N$ is a normalization constant and $a \neq b$. The integral of this weighting function over all $\theta$ is finite and can be performed analytically, yielding

$$
\begin{aligned}
& \int d^{6} \theta W(\theta)= \\
& \frac{16 \pi^{2}}{a} N\left[\frac{(2 c-b)}{b\left(4 c^{2}+b^{2}\right)}+\frac{2 b \tanh ^{-1} \frac{(2 c-b)}{\sqrt{4 c^{2}+b^{2}}}}{\left(4 c^{2}+b^{2}\right)^{3 / 2}}+\frac{2 b \tanh ^{-1} \sqrt{\frac{b^{2}}{4 c^{2}+b^{2}}}}{\left(4 c^{2}+b^{2}\right)^{3 / 2}}-(b \leftrightarrow a)\right] .
\end{aligned}
$$

Henceforth, we will use this result to choose a value for $N$ such that

$$
\int d^{6} \theta W(\theta)=1
$$

Although the form for $W(\theta)$ in Eq. (2.10) is one of many possibilities, it is by far the simplest, smooth function that meets our requirements. Other choices that we have considered do not change our field theoretic results qualitatively. Therefore, in the Lorentz-invariant noncommutative theories that follow, we adopt Eq. (2.10) together with the simple parameter choice $a / 2=b=c=\Lambda^{4}$, where $\Lambda$ sets the scale of the new physics. 


\section{TOY MODELS}

With a suitable weighting function defined, we now consider a few simple applications in toy models. These examples illustrate some of the qualitative features of the noncommutative vertex modification. We consider first noncommutative Yukawa theory, defined by the Lagrangian

$$
\mathcal{L}=\int d^{6} \theta W(\theta)\left[\bar{\psi}(i \not \partial-m) \psi+-\frac{1}{2} \phi\left(\partial_{\mu} \partial^{\mu}+m_{\phi}^{2}\right) \phi+\frac{\lambda}{2}(\bar{\psi} \phi \star \psi+\text { h.c. })\right],
$$

where $\phi$ is a real scalar field. One star product has been removed from each term via integration by parts and the discarding of surface terms. The momentum-space Feynman rule for the fermion-fermion-scalar vertex is

$$
i \lambda \int d^{6} \theta W(\theta) \exp \left[\frac{i}{2} p \cdot \theta \cdot q\right]
$$

where $p$ and $q$ are the four-momenta of the incoming and outgoing fermion. Using the same notation introduced in Section III, we may write the vertex as

$$
\int d^{3} r_{1} d^{3} r_{2} W\left(\vec{r}_{1}, \vec{r}_{2}\right) \exp \left[i \vec{A} \cdot \overrightarrow{r_{1}}+i \vec{B} \cdot \overrightarrow{r_{2}}\right]
$$

where $\vec{A}$ and $\vec{B}$ are functions of the four-momenta given by

$$
\vec{A}=\left(p_{0} \vec{q}-q_{0} \vec{p}\right) / 2 \quad \text { and } \quad \vec{B}=(\vec{p} \times \vec{q}) / 2
$$

Equation (3.3) is symmetric under the interchange of $\vec{A}$ and $\vec{B}$, and reduces to the commutative result, $i \lambda$, in the limit where both are vanishing. We will see, in every application to be discussed in this paper, that either $\vec{A}$ or $\vec{B}$ may be set to zero, by working in an appropriately chosen Lorentz frame. For concreteness, we will set $\vec{B}=0$ and let $\xi \equiv|\vec{A}|$. Then, using the form for $W(\theta)$ in Eq. (2.10), one may express the fermion-fermion-scalar vertex as $i \lambda I_{v}(\xi)$, where

$$
I_{v}(\xi)=-\frac{16 \pi^{2} N}{a \xi} \int_{0}^{\infty} d r_{1} \int_{0}^{r_{1}} d r_{2}\left[r_{2} \sin \left(\xi r_{1}\right)+r_{1} \sin \left(\xi r_{2}\right)\right]\left(e^{-a r_{1} r_{2}}-e^{-b r_{1} r_{2}}\right) e^{-c\left(r_{1}^{2}-r_{2}^{2}\right)}
$$

This integral is finite. One can see that $I_{v}(0)=1$ and $I_{v}(\infty)=0$ by using the fact that

$$
\lim _{\xi \rightarrow 0} \frac{\sin \left(\xi r_{i}\right)}{\xi}=r_{i} \quad \text { and } \quad \lim _{\xi \rightarrow \infty} \frac{\sin \left(\xi r_{i}\right)}{\pi r_{i}}=\delta\left(r_{i}\right)
$$


The integral may be done analytically, though the precise form of the result (given in the appendix) is not particularly enlightening. Qualitatively, $I_{v}(\xi)$ is a function with finite area that drops off quickly as $\sqrt{\xi}$ exceeds the typical scale of noncommutativity, which is set by the choice of the parameters $a, b$, and $c$. In fact, this result could be anticipated from Eq. (3.2), which has the form of a six-dimensional Fourier transform of the weighting function, i.e. $\widetilde{W}\left(\omega_{\mu \nu}\right)$, where the variable conjugate to $\theta$ takes a particular value, $\omega^{\mu \nu}=\left(p^{\mu} q^{\nu}-q^{\mu} p^{\nu}\right) / 4$. It is not hard to see that the integral of $\widetilde{W}\left(\omega_{\mu \nu}\right)$ over all $\omega$ is simply $W(0)$, which is finite. Thus, $\widetilde{W}$ is a function that we would expect to drop off at large values of the argument.

One might wonder at this point whether it would have been easier to specify the unknown function $\widetilde{W}$ in the conjugate space from the start. From a model building perspective, such an approach would be contrived. We have made the reasonable aesthetic choice that the function appearing directly in the Lagrangian Eq. (1.6) be taken as simple as possible. One might hope that this will make an eventual physical interpretation of $W(\theta)$ more transparent. In addition, our weighting function is more easily applied to noncommutative theories, such as those requiring an expansion in $\theta$, that have a more complicated vertex structure than in Eq. (3.2). In any case, the qualitative features of our results depend only on the fact that the weighting function falls off at a well-defined scale.

The simplest process we can study in Yukawa theory is the scattering $f_{1} \bar{f}_{1} \rightarrow f_{2} \bar{f}_{2}$, for two distinct types of fermions. This is purely an $s$-channel process; the two vertices will have noncommutative exponential factors that depend on the factors $p_{1} \cdot \theta \cdot \bar{p}_{1}$ and $p_{2} \cdot \theta \cdot \bar{p}_{2}$. Working in the center-of-mass frame, the spatial vectors $\vec{p}_{i}=-\vec{p}_{i}$ and the quantity that we called $\vec{B}$ in Eq. (3.4) will vanish. The spin-averaged differential cross section is isotropic, and the full scattering cross section is given by

$$
\sigma=\frac{\lambda^{4}}{16 \pi} I_{v}^{4}\left(\xi_{s}\right) \frac{\left(s-4 m^{2}\right)^{2}}{s\left[\left(s-m_{\phi}^{2}\right)^{2}+m_{\phi}^{2} \Gamma_{\phi}^{2}\right]},
$$

where

$$
\xi_{s}=\frac{1}{4} \sqrt{s}\left(s-4 m^{2}\right)^{1 / 2} \text { and } \quad \Gamma_{\phi}=\frac{8 \lambda^{2}}{\pi m_{\phi}^{5}} \xi_{m_{\phi}^{2}}^{3} I_{v}\left(\xi_{m_{\phi}^{2}}\right) .
$$

For $\sqrt{s}$ larger than the typical energy scale set by the parameters $a, b$ and $c$ in $I_{v}$, the cross section is suppressed relative to the commutative result. This behavior is illustrated in Fig. 11. 


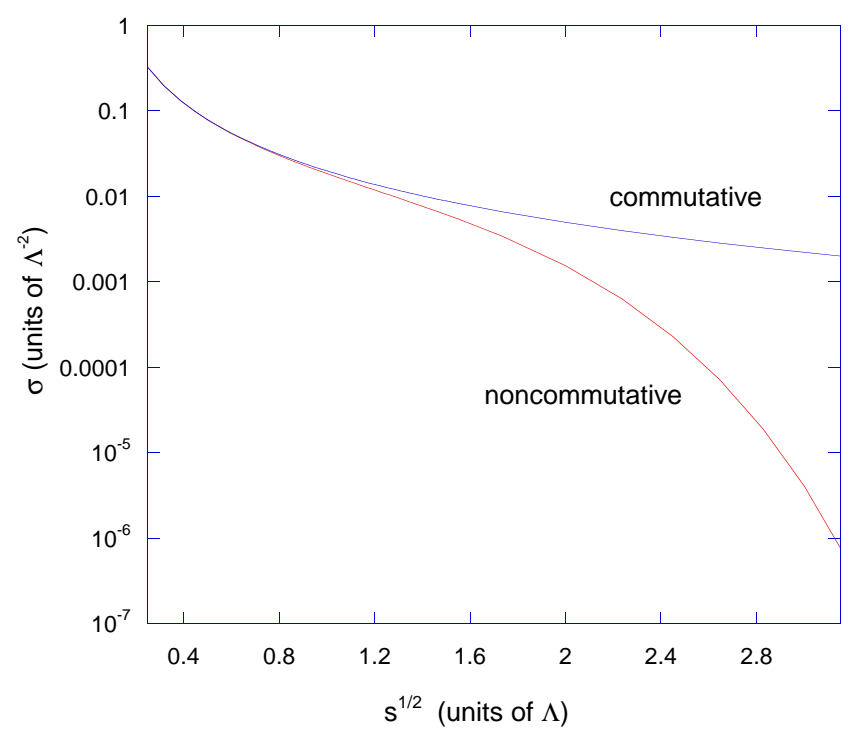

FIG. 1: Difermion production in the Yukawa theory, for the canonical parameter choice $a / 2=b=$ $c=\Lambda^{4}$, and negligible fermion masses.

The modification to the differential cross section is less trivial if one considers processes involving fermions of the same type in the initial and final state. In this case, there are both $s$ - and $t$-channel contributions to the amplitude that receive different noncommutative corrections. Rather than examining $f \bar{f} \rightarrow f \bar{f}$ scattering in the present example, we defer this discussion to the next section where we study Bhabha scattering in QED, which presents a nearly identical calculation.

Instead, to illustrate the dramatic effects that the noncommutative vertex modification can have on angular distributions, we consider tree-level scattering in $\phi^{4}$ theory, defined by the Lagrangian

$$
\mathcal{L}=\int d^{6} \theta W(\theta)\left[-\frac{1}{2} \phi\left(\partial_{\mu} \partial^{\mu}+m_{\phi}^{2}\right) \phi-\frac{\lambda}{4 !}(\phi \star \phi)(\phi \star \phi)\right] .
$$

One star product has been removed from the interaction term via integration by parts. Consider tree-level, $\phi \phi \rightarrow \phi \phi$ scattering in the center-of-mass frame: no example could be simpler in the commutative limit. In the noncommutative case, the momentum-space Feynman rule for the $\phi^{4}$ vertex has the form

$$
\left.i \mathcal{M}=2 \cdot \frac{\lambda}{4 !} \int d^{6} \theta W(\theta)\left[\exp \left[-\frac{i}{2}\left(p_{1} \cdot \theta \cdot p_{2}+p_{3} \cdot \theta \cdot p_{4}\right)\right]+11 \text { other permutations }\right)\right]
$$


where the momenta are outwardly directed. Now assume that $p_{1}$ and $p_{2}$ correspond to incident particles with beam energy $E$ and three-momentum magnitude $p$. One can show that each of the twelve terms in Eq. (3.10) can be written in the same form as Eq. (3.3), with either $\vec{A}$ or $\vec{B}$ identically zero. Thus, using the symmetry of the vertex function under $\vec{A} \leftrightarrow \vec{B}$, one can express each in terms of the function $I_{v}(\xi)$, defined in Eq. (3.5), for some choice of argument $\xi$. We find

$$
i \mathcal{M}=i \frac{\lambda}{3}\left[I_{v}\left(\xi_{1}\right)+I_{v}\left(\xi_{2}\right)+I_{v}\left(\xi_{3}\right)\right]
$$

where

$$
\begin{aligned}
& \xi_{1}=\sqrt{2} E p\left(1+\cos \theta_{0}\right)^{1 / 2}, \\
& \xi_{2}=\sqrt{2} E p\left(1-\cos \theta_{0}\right)^{1 / 2}, \\
& \xi_{3}=p^{2} \sin \theta_{0},
\end{aligned}
$$

and where $\theta_{0}$ is the center-of-mass scattering angle. This leads immediately to the differential cross section

$$
\begin{aligned}
\frac{d \sigma}{d c_{\theta_{0}}}= & \frac{\lambda^{2}}{288 \pi s}\left[I_{v}\left(\frac{\sqrt{s}}{2 \sqrt{2}}\left(s-4 m_{\phi}^{2}\right)^{1 / 2}\left(1+c_{\theta_{0}}\right)^{1 / 2}\right)+I_{v}\left(\frac{\sqrt{s}}{2 \sqrt{2}}\left(s-4 m_{\phi}^{2}\right)^{1 / 2}\left(1-c_{\theta_{0}}\right)^{1 / 2}\right)\right. \\
& \left.+I_{v}\left(\frac{1}{4}\left(s-4 m_{\phi}^{2}\right)\left(1-c_{\theta_{0}}^{2}\right)^{1 / 2}\right)\right]^{2},
\end{aligned}
$$

with $c_{\theta_{0}} \equiv \cos \theta_{0}$. This result reduces to the commutative one at threshold, where the $\operatorname{argument}$ of $I_{v}$ vanishes in each of the three terms. The behavior of this differential cross section for a variety of $\sqrt{s}$ is shown in Fig. 2. The differential cross section is normalized to half the total cross section, which is the value of $d \sigma / d \cos \theta$ at any angle in the limit where the amplitude becomes isotropic. The form of the results in Fig. 2 can be understood by noting that only when $\cos \theta= \pm 1$ are there contributions to the differential cross section that are unsuppressed in the large $\sqrt{s}$ limit. The effect of the tree-level noncommutative vertex modification is therefore to suppress dramatically $\phi \phi$ production transverse to the beam direction. We will see similar modifications to differential cross sections in the more realistic examples to be considered in the next section. 


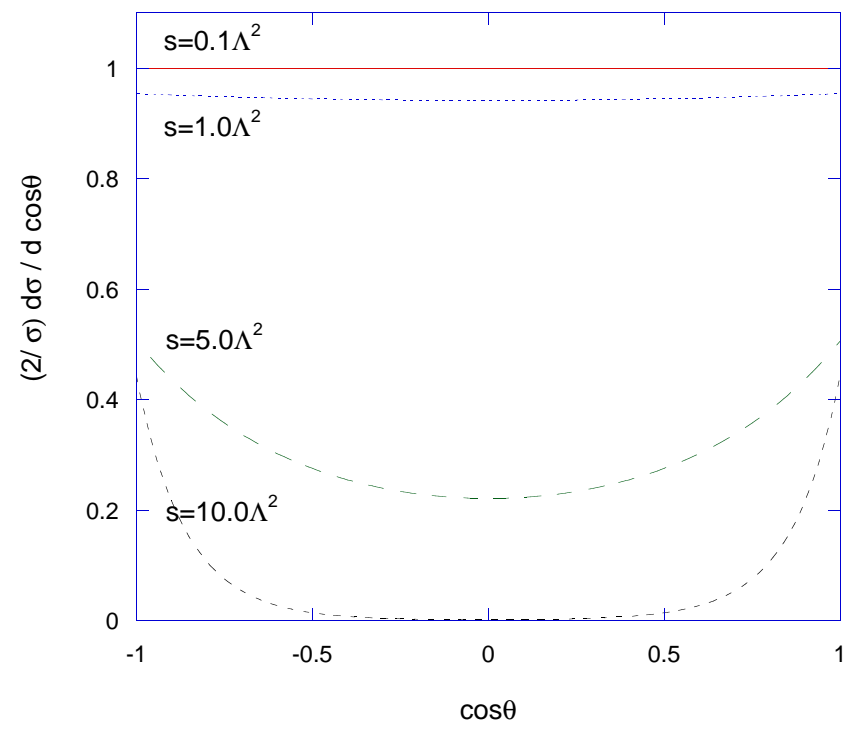

FIG. 2: Differential cross section for two-into-two scattering in $\phi^{4}$ theory, for the canonical parameter choice $a / 2=b=c=\Lambda^{4}$.

\section{NCQED}

We now consider the application of our approach to noncommutative QED. As we mentioned earlier, we focus on the original proposal of Hayakawa 11], which has been studied as a realistic phenomenological theory by a number of authors [5]. The Lagrangian is given by

$$
\mathcal{L}=\int d^{6} \theta W(\theta)\left[\bar{\psi}(i \not \partial-m) \psi-\frac{1}{4} F_{\mu \nu} F^{\mu \nu}+\frac{e}{2}(\bar{\psi} \not A \star \psi+\text { h.c. })\right],
$$

where, as in Sec. III, we have removed one star product via integration by parts and the discarding of surface terms. In this theory, the noncommutative field strength is given by

$$
F_{\mu \nu}=\partial_{\mu} A_{\nu}-\partial_{\nu} A_{\mu}-i e\left[A_{\mu} \stackrel{\star}{,} A_{\nu}\right]
$$

Aside from the modification to the ordinary two-fermion-one-photon vertex, this theory has photon self interactions. Unlike the Hayakawa formulation, however, the three-photon vertex is absent. This interaction is proportional to $\sin \left(p_{i} \cdot \theta \cdot p_{j} / 2\right)$, where the $p_{i}$ are external momenta, but vanishes upon integration against the weighting function $W(\theta)$, which is an even function of $\theta^{\mu \nu}$. Here we focus on dilepton production, Bhabha scattering and diphoton production, processes which are unaffected by the new four-photon vertex at lowest order. 
We may write the Feynman rule for the two-fermion-one-photon vertex as

$$
i e \gamma^{\mu} \int d^{6} \theta W(\theta) \exp \left(\frac{i}{2} p_{1} \cdot \theta \cdot p_{2}\right) \equiv i e \gamma^{\mu} I\left(p_{1}, p_{2}\right),
$$

where $p_{1}$ and $p_{2}$ are the momenta of the incoming and outgoing fermions, respectively. As we will see below, the function $I\left(p_{1}, p_{2}\right)$ may be re-expressed in terms of the vertex integral $I_{v}(\xi)$ given in Eq. (3.5), for an argument $\xi$ that depends on the process in question.

\section{A. Dilepton Production, $e^{+} e^{-} \rightarrow l^{+} l^{-}$}

The matrix element for Bhabha scattering that follows from Eq. (4.3) is:

$$
\begin{aligned}
i \mathcal{M} & =e^{2} \bar{u}\left(p_{3}\right) i \gamma^{\nu} I\left(p_{3}, p_{4}\right) v\left(p_{4}\right) \frac{-i g_{\mu \nu}}{q^{2}+i \epsilon} \bar{v}\left(p_{2}\right) i \gamma^{\mu} I\left(p_{1}, p_{2}\right) u\left(p_{1}\right) \\
& -e^{2} \bar{v}\left(p_{2}\right) i \gamma^{\nu} I\left(p_{2}, p_{4}\right) v\left(p_{4}\right) \frac{-i g_{\mu \nu}}{q^{\prime 2}+i \epsilon} \bar{u}\left(p_{3}\right) i \gamma^{\mu} I\left(p_{1}, p_{3}\right) u\left(p_{1}\right) .
\end{aligned}
$$

Here, $u$ and $v$ represent the momentum-space spinor wave functions for the fermions and antifermions, respectively, and the propagator momenta are given by $q=p_{1}+p_{2}$ and $q^{\prime}=$ $p_{1}-p_{3}$. The four vertex functions $I\left(p_{i}, p_{j}\right)$ can be evaluated using the same approach presented in Sec. III. The functions $I\left(p_{1}, p_{2}\right)$ and $I\left(p_{3}, p_{4}\right)$ are straightforward to simplify since the incoming and outgoing particles are travelling in opposite directions in the centerof-mass frame, so that $\vec{B}=0$ and $\vec{A}=s / 4$. Hence,

$$
I\left(p_{1}, p_{2}\right)=I\left(p_{3}, p_{4}\right)=I_{v}(s / 4)
$$

where $s$ is the usual Mandelstam variable. Here, and throughout this section, we ignore the fermion masses, which are entirely irrelevant given the collider energies of interest (for example, those of a TeV-scale linear collider). Evaluating the remaining functions, $I\left(p_{1}, p_{3}\right)$ and $I\left(p_{2}, p_{4}\right)$, requires a little more effort. Both are Lorentz-invariant functions of the given momenta, so that the result of the integration does not depend on the frame in which it is performed. For example, we can equate $I\left(p_{1}, p_{3}\right)$ to an integral evaluated in a frame where $\vec{B}=0$, simplifying the calculation. In this case, we can work in the $p_{1}$ rest frame, which is 
related to the center-of-mass frame by a boost

$$
p_{1}=\left(\begin{array}{c}
E \\
0 \\
0 \\
p
\end{array}\right) \longrightarrow \Lambda p_{1}=\left(\begin{array}{c}
m \\
0 \\
0 \\
0
\end{array}\right)
$$

where

$$
\Lambda=\left(\begin{array}{cccc}
\gamma & 0 & 0 & -\beta \gamma \\
0 & 1 & 0 & 0 \\
0 & 0 & 1 & 0 \\
-\beta \gamma & 0 & 0 & \gamma
\end{array}\right)
$$

where $\gamma=\left(1-\beta^{2}\right)^{-1 / 2}$ with $\beta=p / E$. On the other hand, $\Lambda p_{3}$ has the spatial components $\left[0, p \sin \theta_{0},-\gamma p\left(1-\cos \theta_{0}\right)\right]$, where $\theta_{0}$ is the center-of-mass scattering angle. After taking the limit $m \rightarrow 0$, the two vectors $\vec{A}$ and $\vec{B}$ become $\left[0,0,-E^{2}\left(1-\cos \theta_{0}\right) / 2\right]=[0,0, t / 4]$ and $[0,0,0]$, respectively. Hence,

$$
I\left(p_{1}, p_{3}\right)=I\left(p_{2}, p_{4}\right)=I_{v}(t / 4)
$$

Note that the simplification of $I\left(p_{2}, p_{4}\right)$ is accomplished using the approach just described, but by evaluating the integral instead in the $p_{2}$ rest frame.

Squaring the matrix element and summing (averaging) over the final (initial) fermion spin states gives

$$
\overline{|\mathcal{M}|^{2}}=2 e^{4}\left[I_{v}^{4}(s / 4)\left(\frac{t^{2}+u^{2}}{s^{2}}\right)+2 I_{v}^{2}(s / 4) I_{v}^{2}(t / 4) \frac{u^{2}}{s t}+I_{v}^{4}(t / 4)\left(\frac{u^{2}+s^{2}}{t^{2}}\right)\right],
$$

where the Mandelstam variables are defined by

$$
s=\left(p_{1}+p_{2}\right)^{2}, \quad t=\left(p_{1}-p_{3}\right)^{2}, \quad u=\left(p_{1}-p_{4}\right)^{2} .
$$

The same results for $e^{+} e^{-} \rightarrow \mu^{+} \mu^{-}$can be obtained easily by discarding the $t$-channel contribution in Eq. (4.9), again ignoring all fermion masses. The spin-averaged, squared matrix element is:

$$
\overline{|\mathcal{M}|^{2}}=2 e^{4} I_{v}^{4}(s / 4)\left(\frac{t^{2}+u^{2}}{s^{2}}\right)
$$


The differential cross section for both processes can be obtained by multiplying the results above by the proper phase space factor:

$$
\frac{d \sigma}{d \cos \theta_{0}}=\frac{\overline{|\mathcal{M}|^{2}}}{32 \pi s} .
$$

Eqs. (4.11) and (4.12) allow us quickly to place a bound on the noncommutativity scale. Noting that at the highest LEP II energies $(\sim 189 \mathrm{GeV})$, the cross section for dimuon production agrees with the stardard model prediction up to $5 \%$ experimental error bars [25], it is reasonable to require that $I_{v}^{4}(s / 4) \gtrsim 0.9$ which leads to the bound

$$
\Lambda>172 \mathrm{GeV} \quad 95 \% \text { C.L. }
$$

This is consistent with the bound on $\Lambda_{N C}$ found in Ref. [25], and quoted in Eq. (1.9]). Note that we cannot adopt the same definition of the noncommutative scale as Ref. [25] because Eq. (1.8) is vanishing for the present choice of weighting function.

\section{B. Diphoton Production, $e^{+} e^{-} \rightarrow \gamma \gamma$}

Diphoton production is particularly interesting in this model since it is different from canonical noncommutative theories (there is no three-photon vertex) and from the theory described in Ref. [25] (there is no two-photon-two-fermion vertex). The matrix element for diphoton production can be written as:

$$
\begin{aligned}
i \mathcal{M} & =e^{2} \bar{v}\left(p_{2}\right) i \gamma^{\mu} I\left(p_{2}, p_{4}\right) \frac{i(\not q+m)}{q^{2}-m^{2}+i \epsilon} i \gamma^{\nu} I\left(p_{1}, p_{3}\right) u\left(p_{1}\right) \epsilon_{\mu}^{*}\left(p_{4}\right) \epsilon_{\nu}^{*}\left(p_{3}\right) \\
& +e^{2} \bar{v}\left(p_{2}\right) i \gamma^{\mu} I\left(p_{2}, p_{3}\right) \frac{i\left(\not q^{\prime}+m\right)}{q^{\prime 2}-m^{2}+i \epsilon} i \gamma^{\nu} I\left(p_{1}, p_{4}\right) u\left(p_{1}\right) \epsilon_{\mu}^{*}\left(p_{3}\right) \epsilon_{\nu}^{*}\left(p_{4}\right) .
\end{aligned}
$$

Here, $p_{1}\left(p_{2}\right)$ is the momentum of the incoming fermion (anti-fermion), while $p_{3}$ and $p_{4}$ are the photon momenta; $u$ and $v$ are the momentum-space spinor wave-functions for the fermion and antifermion, $\epsilon_{\mu}$ represents a photon polarization vector and the propagator momenta are given by $q=p_{1}-p_{3}$ and $q^{\prime}=p_{1}-p_{4}$. Again we may simplify the factors of $I\left(p_{i}, p_{j}\right)$ by boosting to appropriate Lorentz frames. One finds, in addition to Eq. (4.8), that

$$
I\left(p_{1}, p_{4}\right)=I\left(p_{2}, p_{3}\right)=I_{v}(u / 4),
$$




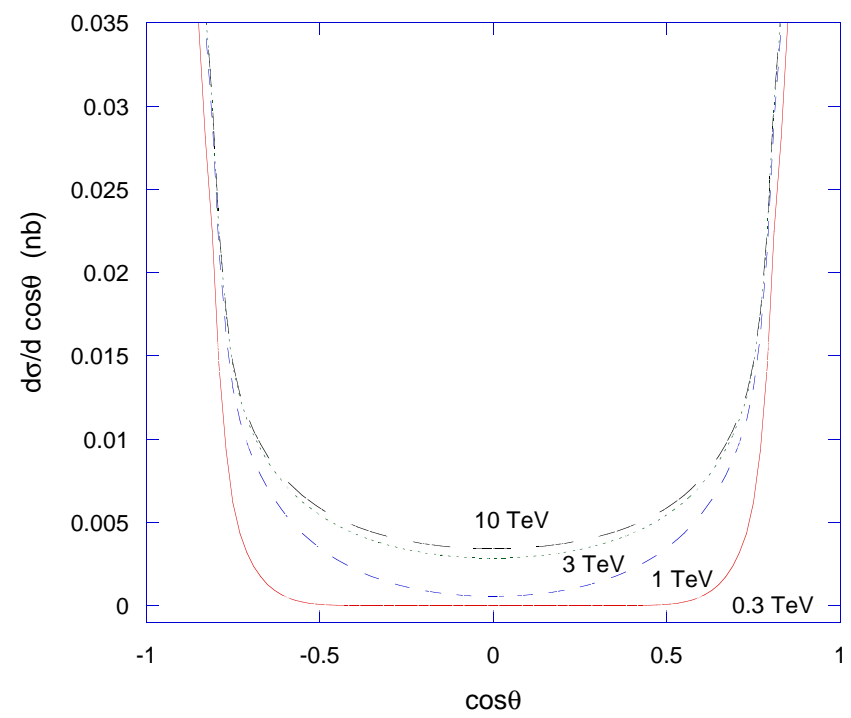

FIG. 3: Differential cross section for diphoton production at a linear collider with $\sqrt{s}=1.5 \mathrm{TeV}$. The curves shown correspond to the canonical parameter choice $a / 2=b=c=\Lambda^{4}$, with $\Lambda=0.1$, 1,3 and $10 \mathrm{TeV}$.

from which it follows that

$$
\frac{d \sigma}{d \cos \theta_{0}}=\frac{\pi \alpha}{s}\left[I_{v}^{4}(t / 4)\left(\frac{u}{t}\right)+I_{v}^{4}(u / 4)\left(\frac{t}{u}\right)\right] .
$$

This cross section shows the same suppression in directions transverse to the beam that we encountered in the case of $\phi^{4}$ theory. This is displayed in Fig. 3, for a linear $e^{+} e^{-}$collider with $\sqrt{s}=1.5 \mathrm{TeV}$, for a range of noncommutative scales $\Lambda$. The standard model result is recovered in the large $\Lambda$ limit, and the figure shows discernable deviations that could, in principle, be extracted via a fit to the eventual data.

\section{Coulomb Potential}

It is interesting to note that the high-momentum modification of the photon-fermionfermion vertex alters the Coulomb potential at short distances. We evaluate the potential by studying the non-relativistic reduction of the $t$-channel scattering amplitude of two distinguishable fermions. For each vertex, $p_{i} \cdot \theta \cdot p_{f}$ is such that $\xi=|\vec{A}|=m|\vec{q}| / 2$ and $|\vec{B}|=\left|\vec{p}_{i} \times \vec{q}\right|$, where $\vec{q}$ is the spatial momentum transfer. We therefore may discard $\vec{B}$ in the nonrelativistic reduction, since it is higher order in $p_{i} / m \ll 1$. In momentum space, the usual form for the 
nonrelativistic potential is modified by an additional factor of $I_{v}^{2}(m q / 2)$ :

$$
V(r)=\frac{e^{2}}{4 \pi^{2} i r} \int_{-\infty}^{\infty} d q \frac{1}{q} \exp (i q r) I_{v}^{2}(m q / 2) .
$$

Normally, one completes this calculation by performing a complex contour integration, with the contour closed in the upper half plane. In this case, one cannot argue that the contour at infinity has a vanishing contribution to the result, due to the additional function in the integrand. It is straightforward to evaluate this integral numerically. Here we will simply state the important qualitative features. At large distances, the Coulomb potential is not altered; this region corresponds to small $q$ for which $I_{v}^{2}(m q / 2) \approx 1$. On the other hand, as one approaches the origin, one finds that $V(r)$ remains nonsingular. Writing Eq. (4.17) as

$$
V(r)=\frac{e^{2}}{4 \pi^{2} r} \int_{-\infty}^{\infty} d q \frac{\sin q r}{q} I_{v}^{2}(m q / 2)
$$

one may use the fact that $\lim _{r \rightarrow 0} \sin q r / r=q$ to see that

$$
V(0)=\frac{e^{2}}{4 \pi^{2}} \int_{-\infty}^{\infty} d q I_{v}^{2}(m q / 2)=\frac{2 \alpha}{\pi m} \int_{-\infty}^{\infty} d \xi I_{v}^{2}(\xi) .
$$

The last integral is finite and is $\mathcal{O}\left(\Lambda^{2} \alpha /(\pi m)\right)$, for our parameter choice $a / 2=b=c=\Lambda^{4}$. Hence, the singularity at the origin is not present, a result that one might attribute to the fuzziness of the noncommutative space. It is important to note that this result is independent of the detailed form of $W(\theta)$ and would be obtained for any weighting function with finite volume in momentum space, since such a function provides an $\mathcal{O}(\Lambda)$ ultraviolet cut-off in Eq. (4.17).

\section{FOUR-FERMION INTERACTIONS}

In the theories that we have considered, the Lorentz-invariant, noncommutative modification to the tree-level vertices leads to a suppression in scattering cross sections at energies above the typical scale of the new physics. It is not unreasonable to guess that, at least in some theories, a violation of unitarity in the commutative limit may be cured by the additional high-momentum suppression of the noncommutative vertex. In this subsection, we will give one example where this is the case, working at tree-level. 
Consider a theory of two distinguishable fermions, $\psi_{1}$ and $\psi_{2}$, with the nonrenormalizable interaction Lagrangian

$$
\mathcal{L}_{i n t}=\frac{1}{M^{2}}\left(\bar{\psi}_{1} \star \psi_{1}\right)\left(\bar{\psi}_{2} \star \psi_{2}\right)
$$

We may assume that the fermions have masses $m_{1}$ and $m_{2}$, though we will only be interested in the behavior of the scattering cross sections at high energies, where these masses are irrelevant.

The possible tree-level, two-into-two scattering processes are fermion-anti-fermion annihilation, and the scattering of fermions and/or antifermions of a different type. With the interaction written as in Eq. (5.1) (again with one star product removed), the relevant differential cross sections in the center-of-mass frame and in the high-energy limit are given by

$$
\begin{aligned}
\frac{d \sigma}{d c_{\theta_{0}}}\left(\psi_{i} \bar{\psi}_{i} \rightarrow \psi_{j} \bar{\psi}_{j}\right) & =\frac{s}{32 \pi M^{4}} I_{v}^{2}\left[s\left(1-c_{\theta_{0}}\right)^{1 / 2} /(2 \sqrt{2})\right] \\
\frac{d \sigma}{d c_{\theta_{0}}}\left(\psi_{i} \psi_{j} \rightarrow \psi_{i} \psi_{j}\right) & =\frac{s\left(1-c_{\theta_{0}}\right)^{2}}{128 \pi M^{4}} I_{v}^{2}\left[s\left(1-c_{\theta_{0}}^{2}\right)^{1 / 2} / 4\right]
\end{aligned}
$$

and

$$
\frac{d \sigma}{d c_{\theta_{0}}}\left(\psi_{i} \bar{\psi}_{j} \rightarrow \psi_{i} \bar{\psi}_{j}\right)=\frac{s\left(1-c_{\theta_{0}}\right)^{2}}{128 \pi M^{4}} I_{v}^{2}\left[s\left(1-c_{\theta_{0}}\right)^{1 / 2} /(2 \sqrt{2})\right],
$$

for $i \neq j$. Clearly, for any $\theta_{0} \neq 0$ or $\pi$, these differential cross sections are suppressed in the large $\sqrt{s}$ limit: the exponential damping of $I_{v}$ above the noncommutative scale wins over the $s / M^{4}$ growth of the commutative result. This conclusion remains unchanged when one integrates over the center-of-mass scattering angle $\theta_{0}$. Provided that the scale of noncommutativity is not far in the ultraviolet, one can prevent an unlimited growth in the total cross section that would exceed the unitarity bounds. To quantify this, one may consider the partial wave decomposition

$$
\left(2 \frac{d \sigma}{d \Omega}\right)^{1 / 2}=\frac{1}{\sqrt{s}} \sum_{J=0}^{\infty}(2 J+1) P_{J}\left(\cos \theta_{0}\right) M_{J}
$$

where the partial wave amplitudes must satisfy the unitarity constraint $\left|M_{J}\right|<1[31]$. In all of the examples above, we find that $s$-wave unitarity provides the tightest bound, which numerically is consistent with the inequality

$$
M \gtrsim 0.4 \Lambda
$$


for our canonical parameter choice $a / 2=b=c=\Lambda^{4}$. Thus, if $M$ is not far below the scale of noncommutative physics, one need not worry that this theory will violate unitarity at tree-level.

\section{CONCLUSIONS}

In this paper, we have revisited a proposal for constructing Lorentz-invariant, noncommutative field theories. Canonical noncommutative field theories involve a Lorentz-violating parameter, while nature shows no indication that Lorentz-invariance is broken. Experimental limits on the amount of Lorentz violation that is tolerable in the low-energy effective theory force the typical energy scale of the noncommutative interactions to be above the center-of-mass energies that can be probed directly in planned collider experiments. This motivated the proposal in Ref. [21] to formulate a Lorentz-conserving alternative to the canonical models.

As we have reviewed earlier, this proposal involves extending the coordinate algebra by promoting the noncommutativity parameter of the canonical theories to a fictitious coordinate. The mapping of this algebra into a field theory is again accomplished through a star product, but the new coordinates are integrated in the action with a weighting factor, which sets the scale of the noncommutative physics. The resulting four-dimensional effective theory remains Lorentz invariant and a function of ordinary coordinates only. Unlike previous phenomenological work based on this approach, we have considered theories in which the prescription described for constructing a Lorentz-invariant noncommutative theory could be implemented and studied without recourse to a low-momentum expansion. In particular, this allows us to study how cross sections behave as typical energies exceed the noncom-

mutative scale, as we have done in both toy models (Yukawa and $\phi^{4}$ theory) and the more realistic case of noncommutative QED for fields in the lepton sector, where the matter have charges \pm 1 or 0 .

Interestingly, we find that cross sections drop quickly as the center-of-mass energy exceeds the noncommutative scale. Roughly speaking, the noncommutative vertex modification smears out the point-like interaction so that at high center-of-mass energies the incoming scatterer sees less and less of the target. Equivalently, the prescription for integrating over 
the fictitious $\theta$ coordinates with the weighting function $W(\theta)$ introduces form-factors in the tree-level vertices of the theory. As we saw in a number of cases, this also has consequences for the angular distributions of scattering processes, since the various contributions to a scattering amplitude with differing angular dependence in the commutative limit may each receive different noncommutative corrections. The modifications to the QED processes of dilepton production, Bhabha scattering and diphoton production were all presented. While

Ref. 25] evaluated the lowest-order effects of noncommutativity on these processes in one version of noncommutative QED (which is appropriate for determining the collider bounds on the model), the results presented here would be useful for a qualitative comparison to the data if, for example, a $1.5 \mathrm{TeV}$ International Linear Collider (ILC) were to discover and study noncommutative physics at a scale comparable to its center-of-mass energy.

Finally, we noted that a modification to a tree-level interaction that suppresses a given cross-section at high center-of-mass energies may alter one's conclusions as to whether the cross section ever violates the constraints from unitarity of the $S$-matrix. We demonstrated this in the case of a simple four-fermion interaction. Since our vertex modification introduces an exponential suppression factor at high $\sqrt{s}$, then a theory whose cross section grows only as a power $\sqrt{s}$ in the commutative limit, will not grow indefinitely in the noncommutative case. Using the constraint from partial-wave unitarity, we obtained a bound on the coefficient of the four-fermion interaction so that the theory would remain unitary for arbitrarily large $\sqrt{s}$. Whether this could be an avenue for constructing a new type of Higgsless theory is an interesting question, but one that first requires a more tractable, all-orders formulation of noncommutative standard model.

\section{Acknowledgments}

We thank Josh Erlich for useful comments. We thank the NSF for support under Grant No. PHY-0456525.

\section{APPENDIX A}

The integrals in Eq. (3.5) may be evaluated, yielding 


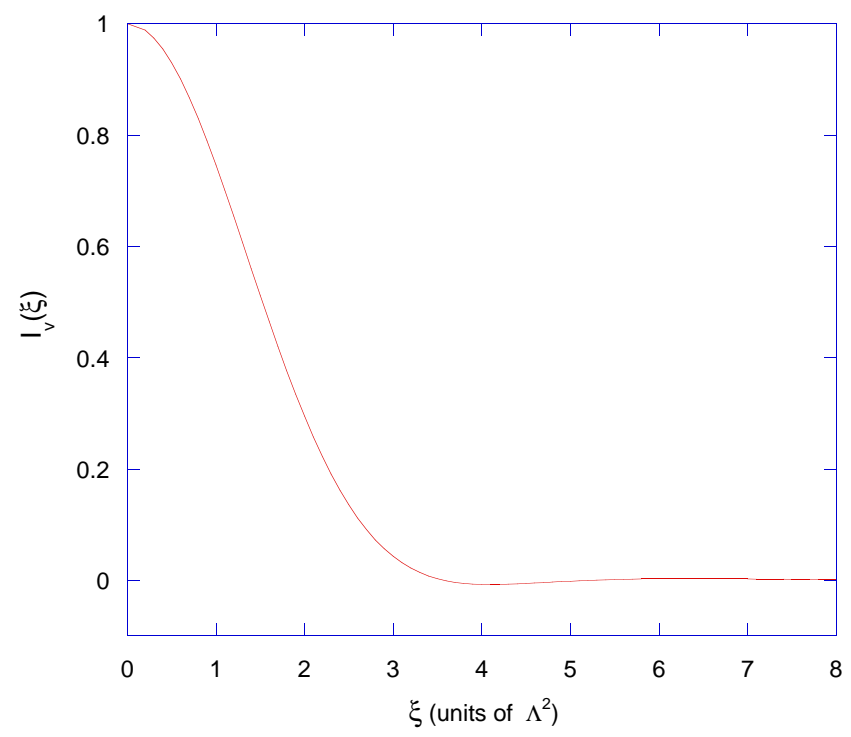

FIG. 4: Plot of the vertex integral function, for the canonical parameter choice $a / 2=b=c=\Lambda^{4}$.

$$
\begin{aligned}
\mathrm{I}_{v}(\xi)= & \frac{16 \pi^{2} N}{a \xi}\left[\frac{\exp \left(\frac{-\xi^{2}}{4 b}\right) \sqrt{\pi} \operatorname{Erfi}\left(\frac{\xi}{2 \sqrt{b}}\right)}{2 c \sqrt{b}}\right. \\
+ & \left.\frac{\xi}{c^{2}} \sum_{0}^{\infty} \frac{n+1}{2 n+1}\left\{\frac{\left(\frac{b}{2 c}\right)\left(1-\frac{b}{2 c}\right)^{2 n+1}+\left(\frac{b}{2 c}\right)^{2 n+2}}{\left(1+\frac{b^{2}}{4 c^{2}}\right)^{2+n}}\right\}_{1} F_{1}\left[2+n, 3 / 2,-\xi^{2} /\left(4 c+b^{2} / c\right)\right)\right] \\
& -(b \leftrightarrow a)]
\end{aligned}
$$

where Erfi is an imaginary error function, and ${ }_{1} F_{1}$ is a confluent Hypergeometric Function of the first kind. This function is plotted in Fig. 4.

[1] For a review, see, R. J. Szabo, Phys. Rept. 378, 207 (2003) arXiv:hep-th/0109162, and references therein.

[2] For a review, see, I. Hinchliffe, N. Kersting and Y. L. Ma, Int. J. Mod. Phys. A 19, 179 (2004) arXiv:hep-ph/0205040, and references therein.

[3] J. Madore, S. Schraml, P. Schupp and J. Wess, Eur. Phys. J. C 16, 161 (2000) arXiv:hep-th/0001203.

[4] I. Hinchliffe and N. Kersting, Phys. Rev. D 64, 116007 (2001) arXiv:hep-ph/0104137;

H. Grosse and Y. Liao, Phys. Lett. B 520, 63 (2001). 
[5] S. Godfrey and M. A. Doncheski, Phys. Rev. D 65, 015005 (2002) arXiv:hep-ph/0108268; arXiv:hep-ph/0111147; N. Mahajan, arXiv:hep-ph/0110148; S. w. Baek, D. K. Ghosh, X. G. He and W. Y. Hwang, Phys. Rev. D 64, 056001 (2001); T. Ohl and J. Reuter, Phys. Rev. D 70, 076007 (2004) arXiv:hep-ph/0406098; H. Grosse and Y. Liao, Phys. Rev. D 64, 115007 (2001); M. Haghighat, M. M. Ettefaghi and M. Zeinali, Phys. Rev. D 73, 013007 (2006) arXiv:hep-ph/0511042; B. Melic, K. Passek-Kumericki and J. Trampetic, Phys. Rev. D 72, 054004 (2005) arXiv:hep-ph/0503133; A. Devoto, S. Di Chiara and W. W. Repko, Phys. Lett. B 588, 85 (2004) arXiv:hep-ph/0401071.

[6] G. Amelino-Camelia, L. Doplicher, S. k. Nam and Y. S. Seo, Phys. Rev. D 67, 085008 (2003) arXiv:hep-th/0109191; G. Amelino-Camelia and S. Majid, Int. J. Mod. Phys. A 15, 4301 (2000) arXiv:hep-th/9907110.

[7] C. S. Chu, B. R. Greene and G. Shiu, Mod. Phys. Lett. A 16, 2231 (2001) arXiv:hep-th/0011241; S. Tsujikawa, R. Maartens and R. Brandenberger, Phys. Lett. B 574, 141 (2003) arXiv:astro-ph/0308169; G. D. Barbosa and N. Pinto-Neto, Phys. Rev. D 70, 103512 (2004) arXiv:hep-th/0407111. G. Calcagni, Phys. Lett. B 606, 177 (2005) arXiv:hep-ph/0406057; Phys. Rev. D 70, 103525 (2004) arXiv:hep-th/0406006.

[8] J. L. Hewett, F. J. Petriello and T. G. Rizzo, Phys. Rev. D 64, 075012 (2001).

[9] M. Chaichian, M. M. Sheikh-Jabbari and A. Tureanu, Phys. Rev. Lett. 86, 2716 (2001).

[10] S. M. Carroll, J. A. Harvey, V. A. Kostelecky, C. D. Lane and T. Okamoto, Phys. Rev. Lett. 87, 141601 (2001) arXiv:hep-th/0105082.

[11] M. Hayakawa, Phys. Lett. B 478, 394 (2000) arXiv:hep-th/9912094.

[12] A. Anisimov, T. Banks, M. Dine and M. Graesser, Phys. Rev. D 65, 085032 (2002) arXiv:hep-ph/0106356.

[13] C. E. Carlson, C. D. Carone and R. F. Lebed, Phys. Lett. B 549, 337 (2002) arXiv:hep-ph/0209077.

[14] A. F. Ferrari, H. O. Girotti and M. Gomes, Phys. Rev. D 73, 047703 (2006) arXiv:hep-th/0510108.

[15] B. Jurco, L. Moller, S. Schraml, P. Schupp and J. Wess, Eur. Phys. J. C 21, 383 (2001) arXiv:hep-th/0104153; X. Calmet, B. Jurco, P. Schupp, J. Wess and M. Wohlgenannt, Eur. Phys. J. C 23, 363 (2002) arXiv:hep-ph/0111115. 
[16] C. E. Carlson, C. D. Carone and R. F. Lebed, Phys. Lett. B 518, 201 (2001) arXiv:hep-ph/0107291.

[17] X. Calmet, Eur. Phys. J. C 41, 269 (2005) arXiv:hep-ph/0401097.

[18] D. Colladay and V. A. Kostelecky, Phys. Rev. D 58, 116002 (1998) arXiv:hep-ph/9809521.

[19] There is a substantial literature on the experimental consequences of the violation of Lorentz invariance. See, for example, R. Lehnert, arXiv:gr-qc/0602073 R. Bluhm, arXiv:hep-ph/0506054, V. A. Kostelecky and M. Mewes, Phys. Rev. D 69, 016005 (2004) arXiv:hep-ph/0309025; R. Bluhm, V. A. Kostelecky, C. D. Lane and N. Russell, Phys. Rev. D 68, 125008 (2003) arXiv:hep-ph/0306190; V. A. Kostelecky and A. G. M. Pickering, Phys. Rev. Lett. 91, 031801 (2003) arXiv:hep-ph/0212382.

[20] M. Chaichian, P. P. Kulish, K. Nishijima and A. Tureanu, Phys. Lett. B 604, 98 (2004) arXiv:hep-th/0408069; F. Koch and E. Tsouchnika, Nucl. Phys. B 717, 387 (2005) arXiv:hep-th/0409012.

[21] C. E. Carlson, C. D. Carone and N. Zobin, Phys. Rev. D 66, 075001 (2002) arXiv:hep-th/0206035.

[22] K. Morita, Prog. Theor. Phys. 111, 881 (2004) arXiv:hep-th/0312080; Prog. Theor. Phys. 110, 1003 (2003) arXiv:hep-th/0309159; Prog. Theor. Phys. 108, 1099 (2003) arXiv:hep-th/0209234.

[23] H. Kase, K. Morita, Y. Okumura and E. Umezawa, Prog. Theor. Phys. 109, 663 (2003) arXiv:hep-th/0212176.

[24] K. Morita, Y. Okumura and E. Umezawa, Prog. Theor. Phys. 110, 989 (2003) arXiv:hep-th/0309155.

[25] J. M. Conroy, H. J. Kwee and V. Nazaryan, Phys. Rev. D 68, 054004 (2003) arXiv:hep-ph/0305225.

[26] M. Haghighat and M. M. Ettefaghi, Phys. Rev. D 70, 034017 (2004) arXiv:hep-ph/0405270.

[27] C. E. Carlson and C. D. Carone, Phys. Rev. D 65, 075007 (2002) arXiv:hep-ph/0112143;

[28] R. Banerjee, B. Chakraborty and K. Kumar, Phys. Rev. D 70, 125004 (2004) arXiv:hep-th/0408197; A. Smailagic and E. Spallucci, J. Phys. A 37, 1 (2004) [Erratumibid. A 37, 7169 (2004)] arXiv:hep-th/0406174; O. Bertolami and L. Guisado, JHEP 0312, 013 (2003) arXiv:hep-th/0306176; S. Ghosh, Mod. Phys. Lett. A 19, 2505 (2004). 
[29] S. Doplicher, K. Fredenhagen and J. E. Roberts, Phys. Lett. B 331, 39 (1994).

[30] H. S. Snyder, Phys. Rev. 71, 38 (1947).

[31] C. Quigg, "Gauge Theories Of The Strong, Weak And Electromagnetic Interactions," Front. Phys. 56, 1 (1983). 